\title{
Physiological Noise Cancellation in fNIRS using an Adaptive Filter Based on Mutual Information*
}

\author{
David Bontrager ${ }^{1}$, Domen Novak ${ }^{2}$, Raphael Zimmermann ${ }^{3}$, Robert Riener ${ }^{2}$, and Laura Marchal-Crespo ${ }^{2}$
}

\begin{abstract}
Functional near-infrared spectroscopy (fNIRS) is a noninvasive optical method that measures cortical activity based on hemodynamics in the brain. Physiological signals (biosignals), such as blood pressure and respiration, are known to appear in cortical fNIRS recordings. Some biosignal components occupy the same frequency band as the cortical response, and respond to the subjects activity. To process an fNIRS signal in a braincomputer interface, it is desirable to know which components of the signal come from cortical response, and which come from biosignal interference. Numerous filtering methods have been proposed to this end with mixed success, possibly because they assume that the cortical and physiological signals combine linearly, or that biosignals do not correlate with subject behavior. Here, we propose an adaptive filter with a cost function based on mutual information to selectively remove information that correlates with blood pressure from the fNIRS signal. The filter was tested with real and simulated data. The real signals were measured on seven healthy subjects performing an isometric pinching task. Cross-correlation and mutual information were employed as performance measures. The filter successfully removed correlations between blood pressure and the fNIRS signal, by an equal or greater amount compared to a traditional recursive least squares adaptive filter. Blood pressure was found to be the most informative signal to classify rest and active periods using linear discriminant analysis. Any task information in the fNIRS signal was redundant to that expressed by blood pressure.
\end{abstract}

\section{INTRODUCTION}

Over the last decades, brain-computer interfaces (BCIs) have emerged as promising solutions to restore lost motor function in a wide range of applications [1]. Their purpose is to translate measurements of neural activity into a control signal that triggers an external device (e.g. a robotic device). The most widely employed methods to noninvasively record brain activation are electroencephalography (EEG), functional magnetic resonance imaging (fMRI), and functional nearinfrared spectroscopy (fNIRS). The use of fNIRS in BCIs has increased substantially in recent years because it is easy to use, relatively tolerant to movement, and can be miniaturized and operated wirelessly [2]. fNIRS probes placed on the scalp measure the amount of near-infrared light absorbed by human tissue between a light source and detector. Given these changes in light intensity, it is possible to calculate the changes in

\footnotetext{
*Research supported by the CHIRP1 ETH Research Grant Cortically-driven Assistance Adaptation during Sensorimotor Training, as well as the National Centers of Competence on Neural Plasticity and Repair and Robotics of the Swiss National Science Foundation.

${ }^{1}$ D. Bontrager is with the Sensory-Motor Systems Laboratory, ETH Zurich, Switzerland

${ }^{2}$ D. Novak, R. Riener, and L. Marchal-Crespo are with the Sensory-Motor Systems Laboratory, ETH Zurich, Switzerland and with the Medical Faculty, Balgrist University Hospital, University of Zurich, Switzerland (phone: +41 4463259 17; e-mail: laura.marchal@mavt.ethz.ch)

${ }^{3} \mathrm{R}$. Zimmermann is with the Biomedical Optics Research Laboratory, University Hospital Zurich, University of Zurich, Switzerland and the Rehabilitation Engineering Laboratory, ETH Zurich, Switzerland
}

oxyhemoglobin (oxyHb) and deoxyhemoglobin (deoxyHb) due to the hemodynamic response to neural activation [3].

fNIRS probes are also sensitive to hemodynamics in the scalp, resulting in the presence of cardiovascular dynamics in the fNIRS signal that often obscure the cortical response [4], [5]. Prominent biosignals that are visible in fNIRS include respiration $(0.2-0.4 \mathrm{~Hz})$ and cardiac activity (blood pressure and cardiac pulse, up to $5.0 \mathrm{~Hz}$ ). These biosignals respond to various tasks, such as isometric pinching [6] and music imagery [7], as part of the autonomic nervous system (ANS) response. The cortical hemodynamics respond at low frequencies (up to $0.2 \mathrm{~Hz}$ ), so band-pass filters can remove faster signals [5] but do not remove in-band biosignal contributions.

Thereby, the biosignals are known to 1) appear in fNIRS [5], and 2) respond to the execution of tasks [6]. The question that follows is, do fNIRS-based BCIs get unique information from cortical activity, or is it entirely redundant to the biosignals' contribution to the fNIRS recording? That is, we wish to see if the fNIRS signals are necessary for such a BCI, or it would be sufficient to use biosignals alone. To find out, any information present in the biosignals must be removed from the fNIRS signal, and an examination made of where task information resides.

A number of methods have previously been applied to remove in-band noise. Among them are principal component analysis (PCA) filters [8], [9], Kalman filters [10], [11], transfer function (TF) models [12], and non-state space based adaptive filters [11]. PCA filters are unconvincing because they rely on the assumption that single phenomena lead to single eigenvectors [8]. The Kalman filter and transfer function approaches impose squared error as the optimization criterion. Additionally, the Kalman filter approach assumed a static baseline of cerebral activity [10], which is not the case [5]. In light of these shortcomings, non-state space based adaptive filters (AFs) are the most appealing option. There is a strong theoretical foundation for these filters and they are able to handle non-stationarity and other peculiarities of real data [14]. A common and well known AF uses the recursive leastsquares (RLS) algorithm [16], [17], since it offers excellent performance in a changing environment.

Due to the nature of the squared error optimization criterion employed in most $\mathrm{AFs}$, least-squares filters are insensitive to potential distortions [18], implying that ANS contributions to fNIRS are minimally distorted copies of peripheral recordings of the ANS signals. This only allows them to remove components that are linearly correlated with the peripherally recorded biosignals, and thus do not address other signal components that represent shared information. As an alternative to least squares approaches, mutual information (MI) is a metric of interdependence between two signals that is sensitive to any linear or nonlinear relationship. It directly addresses 
information content of the signals as determined by statistical dependencies.

Here, we describe an adaptive filter with an MI cost function to selectively remove the blood pressure component from recorded fNIRS. This is the first instance we are aware of that applies MI-based filtering to fNIRS analysis. An RLS adaptive filter was also employed to compare results to an established method. Performance of both filters was compared to validate the MI filter's ability to reduce correlations between fNIRS and BP. Blood pressure and both pre- and post-AF fNIRS signals were then used in a linear discriminant analysis (LDA) classifier to detect rest and active periods in order to determine which signal was most informative to the task.

\section{METHODOLOGY}

\section{A. Data Acquisition}

1) Real data: Data were used from Zimmermann et al's study [19]. Seven healthy subjects performed an isometric pinching task with the right hand while lying in a supine position. fNIRS, blood pressure (BP), and respiration (BR) signals were simultaneously recorded. The task protocol contained fifteen $20 \mathrm{~s}$ pinch periods, each pinch period followed by a rest period of random duration between $15 \mathrm{~s}$ and $24 \mathrm{~s}$. The measurement protocol is described in detail in Zimmermann, et al [19].

Two fNIRS probes (Adult flexible sensor ${ }^{\mathrm{TM}}$, ISS, Inc.) were positioned on the left premotor and primary motor cortices, respectively. Cortical data were obtained with a tissue oximeter (Oxiplex $\mathrm{TS}^{\mathrm{TM}}$, ISS, Inc.) at a sampling frequency $\left(F_{s}\right)$ of $50 \mathrm{~Hz}$. The fNIRS probe's source-detector distances were: $2,2.5,3.5$, and $4 \mathrm{~cm}$. Biosignals were collected with a biosignal amplifier (g.USBamp@), g.tec) at $F_{s}=600 \mathrm{~Hz}$. BP was acquired with a noninvasive blood pressure monitor (CNAP monitor $500^{\mathrm{TM}}$, CNSystems). BR was acquired with a thermistor-based respiratory flow sensor (SleepSense(R)).

2) Synthetic fNIRS Signal: A synthetic fNIRS test signal (fNIRS $\mathrm{fS}_{\mathrm{TS}}$ ) was generated to test filters on known signals. The hemodynamic response function (HRF) was modeled as the difference between two gamma functions, following Worsely, et al [20]. The HRF is a model of how cortical hemodynamics respond to tasks, providing a noise-free neural signal. It was then convolved with a binary activation signal taken from subject 6's task protocol (the activation signal equals 1 during pinch periods and 0 otherwise). Gaussian noise $(\sigma=0.1, \mu=0)$ and simulated BP (primary frequencies: 0.1 , $1.0 \mathrm{~Hz})$ and BR $(0.2 \mathrm{~Hz})$ signals were added as noise, created with Zhang, et al's model [15]:

$$
\mathrm{fNIRS}_{\mathrm{TS}}=\mathrm{HRF}+\mathrm{BP}+\mathrm{BR}+\text { Gaussian noise }
$$

Equation (1) does linearly combine the signals, but this allows us to test if the filter can remove information in one signal and leave intact uncorrelated information in the signal.

\section{B. fNIRS Signal Processing}

Algorithms were implemented in MATLAB 2012b (The MathWorks, Inc.). First, all signals were band-pass filtered at
$0.019-0.2 \mathrm{~Hz}$ to remove slow drift across trials and signals faster than the cerebrovascular response [19] $(0.019=1 / 54$ $\mathrm{Hz}$, for $54 \mathrm{~s}$ trials). Next, an adaptive filter (AF) was used to remove in-band BP components from the fNIRS signals. Unless stated otherwise, all signals discussed hereafter are band-pass filtered.

1) Adaptive Filters: An AF update rule (Fig. 1) adjusts the $L$ filter coefficients in $K(t)=\left[k_{1}, \ldots, k_{L}\right]$ at every time step $t$ based on the error between noise reference $n(t)$ and the filter's estimate of the noise reference, $\hat{n}(t)$. The cost function is the quantity the filter minimizes or maximizes by adjusting its coefficients. The cost function can be chosen based on the task at hand, but minimizing a squared error term is most common [14]. The filter estimates the desired output $\hat{d}(t)$ as the input $x(t)$ without contributions from $n(t)$. If $\mathbf{x}(t)=[x(t), x(t-$ $1), \ldots, x(t-L+1)]^{T}$, then the output is calculated as $\hat{d}(t)=$ $x(t)-K(t) \mathbf{x}(t)[14]$.

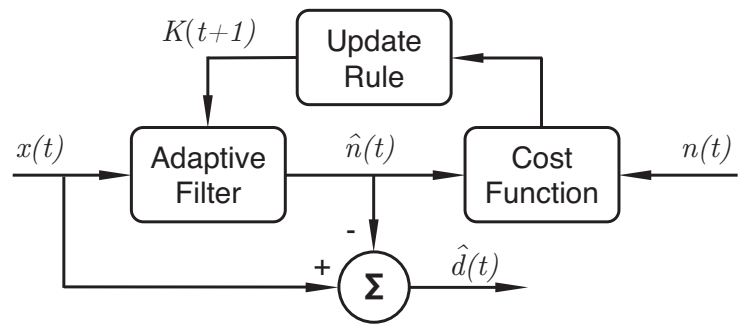

Fig. 1. Adaptive filter schematic.

RLS AFs can converge to the Wiener solution given enough time, meaning they are squared error optimal [14]. However, squared error is insensitive to nonlinear relationships and distortions in signal shape that can still maintain some shared information [18].To overcome these limitations, the mutual information was investigated as a potential alternative to the traditional squared error cost function.

2) Mutual Information: Traditional machine learning methods have been extended to use information-theoretic quantities such as MI [22]. MI is a measure of how much two random variables deviate from being statistically independent of each other, and is sensitive to both linear and nonlinear relationships. The mutual information of random variables $X$ and $Y$ is [21]

$$
\operatorname{MI}(X, Y)=\sum_{x} \sum_{y} p(x, y) \log \frac{p(x, y)}{p(x) p(y)}
$$

for probabilities $p(x)$ and $p(y)$ of $X$ and $Y$, respectively, and their joint probability $p(x, y)$. If $\mathrm{MI}=0$, then $X$ and $Y$ are independent. As the dependence between $X$ and $Y$ increases, so does MI.

Probability densities are notoriously difficult to estimate for few data [22]. Given short, noisy data, kernel density estimators (KDEs) were found to give densities that provide the most accurate estimate of MI [23]. The densities used in the MI calculation were estimated using KDEs with a Gaussian kernal. 


\section{A Mutual Information Cost Function}

Our goal is to minimize the biosignals' presence in the fNIRS signal. A cost function of MI between the filter's output and the noise reference explicitly addresses that shared information. While MI has previously been used in cost functions, our approach uniquely 1) applies it to fNIRS analysis, and 2) explicitly minimizes MI between output and noise reference in contrast to using other quantities. If $K^{*}$ is the optimal filter for cost function $J(K)$, and

$$
\begin{aligned}
J(K) & =\operatorname{MI}(\hat{d}(t), n(t)), \text { then } \\
K^{*} & =\underset{K}{\arg \min } J(K) .
\end{aligned}
$$

To find the optimal coefficients, the update rule uses the standard gradient descent algorithm, which finds $K^{*}$ by adjusting each coefficient of $K$ according to the gradient of $J$ in terms of that coefficient [14], with the adjustment scaled by learning rate $\mu$ :

$$
k_{i}(t+1)=k_{i}(t)-\mu \frac{\partial}{\partial k_{i}} J(K)
$$

Equation (5) can be derived analytically, with each probability in (2) estimated by a KDE. Note that, while the cost function is sensitive to nonlinearities, $K$ is still a linear filter.

\section{Evaluation}

1) Filter Performance: Filter performance was assessed by comparing the cross-correlation function $\left(C_{x y}\right)$ and MI between BP and fNIRS before and after adaptive filtering, for both real ("REAL") and simulated ("SIM") data. The $C_{x y}$ and MI between the simulated data and the theoretical HRF were also calculated, giving a measure of how closely the filtered signal matched the underlying response. Both MI and $C_{x y}$ were reported as the maximum value calculated across up to $10 \mathrm{~s}$ of lag in either direction, to allow for delays between the signals.

2) Classifier Performance: LDA was used to classify rest and active pinching periods on a single-trial basis, using the real fNIRS signal both before and after adaptive filtering, and the BP. For a given pinching or rest period as defined by the task protocol, the mean of the signal of interest was calculated, and given to the classifier as the classification feature. The mean accuracy (Acc.) across all subjects was used to assess the classifier performance, with each subject's accuracy calculated as the mean across a four-fold cross-validation. To reject the hypothesis at the $p=0.05$ significance level that the classifier peformed by chance, the accuracy threshold was calculated as $64.1 \%[6]$.

\section{RESULTS}

Filter results are in Table I, reporting means and standard deviations of the real data's results $(n=7)$. The first column shows the signals between which the correlation is calculated (e.g. "SIM-BP" is between simulated data and BP). Correlations with the HRF are reported for simulated data because the de-noised output is known, and thus it can measure how close to the true signal the filter output is. "Raw Data" is bandpass filtered, "RLS-filtered" further has been processed with an RLS filter, and "MI-filtered" with the MI filter. $p$-values are calculated with paired t-tests between the MI values for all subjects. The goal of the filter is to decrease correlation between BP and fNIRS (remove BP information from the fNIRS signal), and ideally also increase correlation between fNIRS and the HRF (SIM data only).

The MI filter clearly decreased MI and $C_{x y}$ for both real and simulated data with BP. The RLS filter also decreased correlations, but less substantially. For real data, we see that the reduction in MI was significant for the MI filter but not the RLS filter (reported $p$-values are the result of paired ttests of the MI between REAL and BP data for pre-AF compared to post-AF values). Additionally, the RLS filter did little to correlations between SIM and HRF, while the MI filter increased both MI and $C_{x y}$. Fig. 2 shows the simulated fNIRS, and the results for filtering with RLS and MI filters. The pristine HRF is shown in black on each trace.

Table II reports the mean accuracy of the classifier for the different signals. BP was included for comparison due to its high correlation with the task. BP has the highest rate, and is the only signal to classify above chance level. Based on paired t-tests, none of the signals classify with significant difference from each other at the $p=0.05$ level, though MI-filtered classification differs from BP classification with marginal significance.

\section{TABLE I. FILTER PERFORMANCE}

\begin{tabular}{|l||l||c||c||c|}
\hline Signals & Metric & Raw Data & RLS-filtered & MI-filtered \\
\hline SIM-BP & MI & 0.39 & 0.31 & 0.12 \\
& $C_{x y}$ & 0.15 & 0.04 & 0.06 \\
\hline SIM-HRF & MI & 0.51 & 0.48 & 0.76 \\
& $C_{x y}$ & 0.37 & 0.38 & 0.63 \\
\hline REAL-BP & MI & $0.21 \pm 0.15$ & $0.10 \pm 0.06$ & $0.06 \pm 0.02$ \\
& $C_{x y}$ & $0.36 \pm 0.19$ & $0.21 \pm 0.07$ & $0.13 \pm 0.05$ \\
$p$-value & (MI) & & 0.104 & 0.036 \\
\hline
\end{tabular}

TABLE II. Classifier Results

\begin{tabular}{|l||l||c||c||c|}
\hline & Raw Data & RLS-filtered & MI-filtered & BP \\
\hline Acc. $(\%)$ & $60.4 \pm 30.9$ & $56.0 \pm 21.7$ & $50.7 \pm 13.7$ & $75.9 \pm 24.3$ \\
\hline$p$-value & w/raw data & 0.674 & 0.264 & 0.383 \\
& w/BP & 0.143 & 0.056 & \\
\hline
\end{tabular}

\section{DICUSSION}

\section{A. Filtering and Classification}

The MI filter was more successful than the RLS filter at reducing correlations between fNIRS and BP signals. This gives rise to the following speculations. First, that local vascular regulation through the body introduces a distorted or nonlinear relationship between the peripherally recorded $\mathrm{BP}$ and its contribution to the fNIRS recording. This would decrease the RLS filter's ability to remove all BP contributions, while the MI filter could still find a different solution that accomplishes the goal.

Second, it could mean that the BP component in fNIRS is in fact a linearly added version of the peripheral BP recording, 


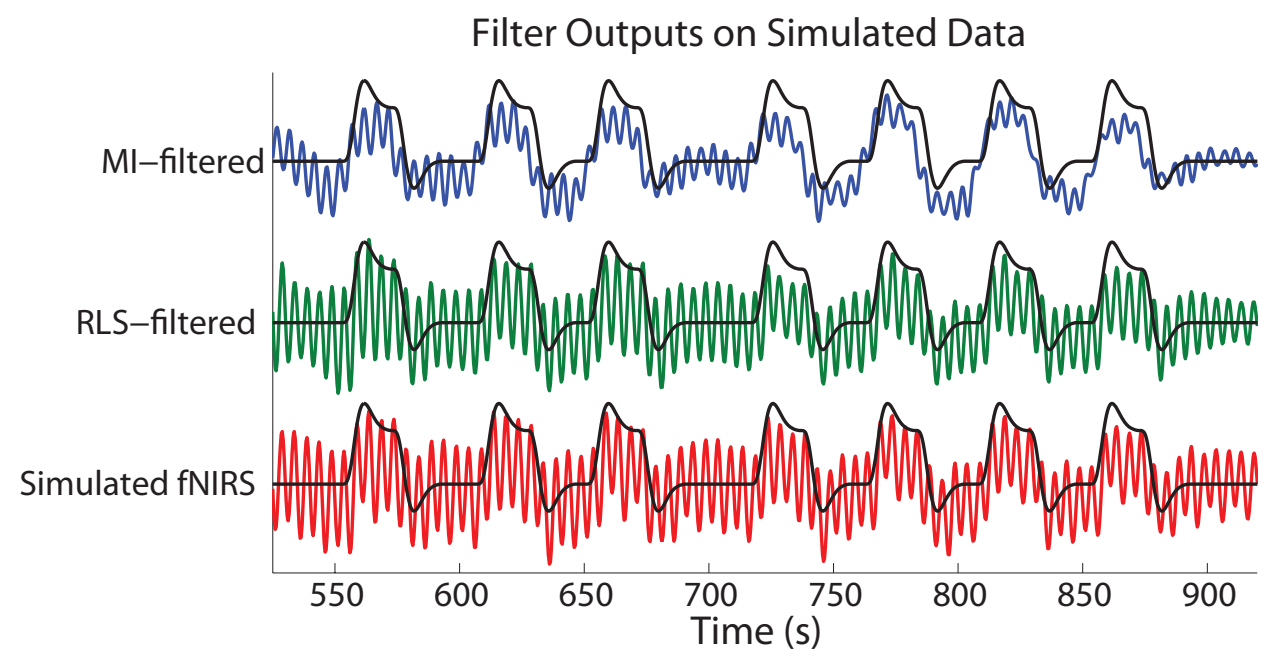

Fig. 2. Filtering simulated fNIRS data.

but that the cortical response within the fNIRS signal correlates strongly with BP's response to the task. In this case, the MI filter would see all task information in the fNIRS recording as correlated with BP and try to remove it, regardless of whether the information is of cardiovascular or cerebrovascular origin. That is, if cortical task information is entirely redundant to BP task information, the filter removes both.

The classifier results do not recommend one alternative over the other - their strongest implication is that, when the fNIRS signal contains no BP information (post-AF), it is unable to classify results.

\section{B. Information Content of Signals}

Despite all the previous work put into separating cortical and ANS contributions to fNIRS recordings, little has been done to disentangle information content of the various signals or provide insight into redundancy among signals [25]. In the context of wanting to use fNIRS as a BCI input, one should know if it contains unique information. If $\mathrm{BP}$ is also used as an input to the BCI, as done by Marchal-Crespo et al [6], the fNIRS appears to carry no task information that is not already contributed by BP. For a binary classifier, BP is a suitable choice if one wishes to have a single control signal. To classify in higher dimensions (e.g. "move right" vs. "move left" vs. "rest"), cortical fNIRS once again becomes attractive due to the ability to record from both hemispheres, which could give a differential response for the three conditions, while BP is limited to binary activation.

\section{CONCLUSIONS}

We have shown that an adaptive filter with a mutual information cost function successfully reduces correlations between fNIRS signals and blood pressure, by a greater amount than a traditional RLS adaptive filter. After blood pressure information is removed from fNIRS, the filtered fNIRS appears to provide little additional control information in a binary activation task. Therefore, at least in simple binary BCI applications, the same information driven by fNIRS could be easily obtained from blood pressure signals.

\section{REFERENCES}

[1] J. R. Wolpaw, N. Birbaumer, D. J. McFarland, G. Pfurtscheller, and T. M. Vaughan, Brain-computer interfaces for communication and control, Clinical Neurophysiology, vol. 113, no. 6, pp. 767 791, 2002.

[2] T. Muehlemann, D. Haensse, M. Wolf, Wireless miniaturizes in-vivo near infrared imaging, Optics Express, vol. 16, pp. 10323-10330, 2008.

[3] A. Villringer, B. Chance, Non-invasive optical spectroscopy and imaging of human brain function, Trends in Neurosciences, vol. 20:435442, 1997.

[4] S.G. Diamond, T.J. Huppert, V. Kolehmainen, M.A. Franceschini, J. P Kaipio, S.R Arridge, and D.A. Boas. Dynamic physiological modeling for functional diffuse optical tomography. Neuroimage, vol.30, no.1, pp.88-101, 2006

[5] C. Elwell, R. Springett, E. Hillman, and D. Delpy, Oscillations in cerebral haemodynamics. Advances in experimental medicine and biology, vol.471, pp.57-65, 1999.

[6] L. Marchal-Crespo, R. Zimmermann, O. Lambercy, J. Edelmann, M.C. Fluet, R. Riener, M. Wolf, R. Gassert,Motor Execution Detection Based on Autonomic Nervous System Responses, Physiological Measurement, vol. 34, no. 1, pp. 35-51, Jan 2013.

[7] T.H. Falk, M. Guirgis, S. Power, T. Chau. Taking NIRS-BCIs Outside the Lab: Towards Achieving Robustness Against Environment Noise. Neural Systems and Rehabilitation Engineering, IEEE Transactions on, vol. 19, no.2, pp. 136-146, 2011.

[8] Y. Zhang, D.H. Brooks, M.A. Franceschini, and D.A. Boas. Eigenvector-based spatial filtering for reduction of physiological interference in diffuse optical imaging. Journal of biomedical optics, vol.10, no.1, pp.011014-01101411, 2005.

[9] A. Chaddad. Brain Function Diagnosis Enhanced Using Denoised fNIRS Raw Signals. J. Biomedical Science and Engineering, vol.7, num.4, pp. 218-227, 2014.

[10] S.G. Diamond, T.J. Huppert, V. Kolehmainen, M.A. Franceschini, J.P. Kaipio, S.R. Arridge, and D.A. Boas. Physiological system identification with the kalman filter in diffuse optical tomography. In Medical Image Computing and Computer-Assisted Intervention -MICCAI 2005, pp.649-656. Springer, 2005.

[11] A. F. Abdelnour and T. Huppert. Real-time imaging of human brain function by near-infrared spectroscopy using an adaptive general linear model. NeuroImage, vol.46, no.1,pp.133-143. Elsevier, 2009.

[12] G. Bauernfeind, C. Boeck, S.C. Wriessnegger, G.R. Mueller-Putz. Physiological Noise Removal from fNIRS Signals. Biomedical Engineering / Biomedizinische Technik. ISSN (Online) 1862-278X, September 2013.

[13] T. Yamada, S. Umeyama, and K. Matsuda, Separation of fnirs signals into functional and systemic components based on differences in hemodynamic modalities. PloS one, vol.7, no.11, 2012. 
[14] P. Diniz, Adaptive Filtering, Algorithms and Practical Implementation. 4th ed. NY: Springer, 2013.

[15] Q. Zhang, E.N. Brown, G.E. Strangman, Adaptive filtering for global interference cancellation and real-time recovery of evoked brain activity: a monte carlo simulation study, J. Biomed. Opt., vol. 12, no. 4, Jul-Aug 2007

[16] F. C. Robertson, T. S. Douglas, and E. M. Meintjes, Motion Artifact Removal for Functional Near Infrared Spectroscopy: A Comparison of Methods, IEEE Trans. Biomedical Engineering, vol. 57, no. 6, pp. 13771387, June 2010

[17] M. Aqil, K. S. Hong, M. Y. Jeong, and S. S. Ge, Cortical brain imaging by adaptive filtering of NIRS signals, Neuroscience Letters, vol. 514, no. 1 , pp. $35-41,2012$

[18] Z. Wang and A. C. Bovik, Mean squared error: love it or leave it? A new look at signal fidelity measures, Signal Processing Magazine, IEEE, vol. 26, no. 1, pp. 98-117, 2009.

[19] R. Zimmermann, L. Marchal-Crespo, O. Lambercy, M.C. Fluet, R. Riener, M. Wolf, R. Gassert, Detection of motor execution using a hybrid fNIRS-biosignal BCI: a feasibility study, J. of NeuroEngineering and Rehabilitation, vol. 10, no. 4, 2013.

[20] K.J. Worsley, C.H. Liao, J. Aston, V. Petre, G.H. Duncan, F. Morales, and A.C. Evans, A general statistical analysis for fmri data, NeuroImage, vol. 15, no. 1, pp. 1-15, 2002.

[21] T. M. Cover and J. A. Thomas, Elements of Information Theory. John Wiley and Sons, 2012.

[22] J. C. Principe, D. Xu, and J. Fisher, Information theoretic learning, Unsupervised Adaptive Filtering, 1, pp. 265-319, 2000.

[23] S. Khan, S. Bandyopadhyay, A. R. Ganguly, S. Saigal, D. J. Erickson III, V. Protopopescu, and G. Ostrouchov, Relative performance of mutual infomration estimation methods for quantifying the dependence among short and noisy data, Physical Review E, vol. 76, no. 2, pp. 026209, 2007.

[24] K. Hlavackova-Schindler, M. Palus, M. Vejmelka, and J. Bhattacharya,
Causality detection based on information-theoretic approaches in time series analysis, Physics Reports, vol. 441, no. 1, pp. 1-46, 2007.

[25] T. Yamada, S. Umeyama and K. Matsuda. Separation of fNIRS signals into functional and systemic components based on differences in hemodynamic modalities. PLOS ONE 7(11): e50271, 2012. 